\title{
SOCIAL NETWORKS AND WEB ONTOLOGY'S AS PERFECT COUPLE FOR KNOWLEDGE MANAGEMENT TOOL/SYSTEM
}

\author{
${ }^{1}$ Florim Idrizi, ${ }^{2}$ Uran llazi, Florin Asani ${ }^{3}$ \\ 1 (Department of IT, Faculty of Natural Sciences and Mathematics, State University of Tetova \\ e-mail: florim.idrizi@unite.edu.mk ) \\ 2 (Department of IT, Faculty of Natural Sciences and Mathematics, State University of Tetova \\ e-mail:uran.ilazi@gmail.com) \\ 3 (Department of IT, Faculty of Natural Sciences and Mathematics, State University of Tetova \\ e-mail:florin.asani@unite.edu.mk )
}

\section{ABSTRACT}

The social networks have become a main tool of the XXI century for personal presentation and promotion, connectivity with others, belonging to same ideology, area of expertise or culture. Beside all the "exploitations" made to the social networks, lets pose the question: Are social networks and social network medias pushed to their limits, are they manageable and do they give as maximal possibilities in using, managing and having all the knowledge "stuffed" in there and using it to benefit of our research? Combining the social networks with semantic web can enhance the process of education and research.

Keywords :Social networks;semantic web

\section{Council for Innovative Research}

Peer Review Research Publishing System

Journal: INTERNATIONAL JOURNAL OF COMPUTERS \& TECHNOLOGY

Vol.14, No. 3

www.ijctonline.com, editorijctonline@gmail.com 


\section{INTRODUCTION}

\subsection{Classic type of social network}

The social networks are online platforms, offering their users, mainly to communicate on-line, present them self to the world, connect to others with same interests or being related to the other users in any other way.

In this chapter, will be presented an snapshot of possible social network, based on usual functionalities offered by most of the common social network platforms (facebook, linkedln,google+, academia.edu etc)

The first step in entering the social network is to create a profile, choose his interests and give him the possibility to enter more information about himself, in order of giving clear definition about his personality, area of expertise, life or research experience, in order to make it possible to the network, to suggest him the accurate users corresponding to his interest and making it possible to be found by the right peoples of his interests.

Most used and one of the basic functionalities is the "SEARCH" function. Most common possibilities offered, are users to connect, pages to follow, communities to attend, events, documents, discussions and similar.

The feed: is the live stream of what is being posted, the possibility to post, comment and being up to date with your group of interests.

Post element : with this element, users are in able to post updates, photos, videos, links, polls articles documents etc. With every post that the user makes, he is in able to choose: interest (category this falls in), visibility (only me, friends, public, custom), location, associate (or most commonly used as tag)

Filter: This element will is part of the feed, where the user is in able to filter what he sees by: All, My posts, Trending News, Latest News, Friends, Pages, Groups and More (this will be a dropdown where we will filter the posts according the interests they fall in. The interests presented will be the ones that the user chose during registration or afterwards)

Pages: This can be considered as profile on its own. User can create, manage and admire pages on the platform. By creating a page, he can switch his account and act as the page itself (this is just a suggestion). Usually the page represents an organization or company or product he likes to present and promote via gathering admires and keeps them up to date. The page will have a menu of content on its own: About, Members, Events, Media Corner.

Maybe it's just about enough for our snapshot of our semantic WEB based social network concept.

\section{SEMANTIC WEB AND ONTOLOGY'S}

The semantic WEB as a technology is to be defined as a bridge between the way as humans think and the way as the machines can understand us, in other words, the machine can understand the meaning of our research and deliver us the exact match of the requested data(for the machine) or information(for the humans)[1].

To achieve this goal, in the semantic web are evolved few methods and tools and frameworks such as RDF, RDFS,SPARQL and OWL[2, 3]

RDF and RDFS - Resource Description Framework and RDF Schema language. RDF addresses one fundamental issue in the Semantic Web: managing distributed data. All other SemanticWeb standards build on this foundation of distributed data. RDF relies heavily on the infrastructure of the Web, using many of its familiar and proven features, while extending them to provide a foundation for a distributed network of data[4]. While the RDF itself creates a graph structure to represent the data, the RDFS gives guidelines on how to use the graph the proper way.

The ontology's purpose is to study the existing things in a domain identify and defining their classification [5] in a matter that will be understandable for the machine processor. That definition is expressed with help of the OWL.

One of the great powers of the Semantic Web is that information that has been specified by one person in one context can be reused either by that person or by others in different contexts. There is no expectation that the same source who defined the roster of players will be the one that defines the role of the agents or of the bank accounts. If we want to use information from multiple sources together, we need a way to express concepts from one context in terms of concepts from the other. In OWL, this is achieved by having a facility with which we can describe new classes in terms of classes that have already been defined[4]

\section{SOCIAL NETWORK AND SEMANTIC WEB}

The normal classical social network platforms, offer possibilities of managing the way you post, visibility of your posts, posts of your friends and colleagues in some structured way, which is manageable and reusable information until given amount of data. After that limit is reached, the posted data is reusable just for a given time, when it's still actual. After new data gets layered, possibility of finding any specific information related to given topic becomes near to impossible.

To avoid the unstructured data, the semantic web [1] can be placed behind the interests, which are mentioned at the first chapter of this paper.

Reviewing all the elements or functionalities of a social network, we can reveal the ontology's behind the actors and their contributions. 
The first step, creating profile and choosing interest, the interest itself require ontology definitions. By that, we are defining if the person is scientist, $\mathrm{PhD}$. Attendant, is administration worker in a scientific institution or is simply student. If is a scientist, will be part of the scientific direction, field of research and.

Ph.D attendant will be interested in the postgraduate research, field of research and going further to details, and even define its connection to his mentor.

Administrative worker, will chose the department as interest, his field of work group of participants covering or being responsible for.

Those three elements should serve as overview for the possibilities of the semantic web with an social network platform.

\section{POSSIBLE SCENARIOS}

The student want to see the professors and their lesions eligible for his study, according to his itinerary, lesions and predefined preferences of time and ETC credits wanting to achieve for the actual semester.

The professor targets the students attending his lectures, and providing them with materials, papers and possible groups of interest which would be beneficiary for the lecture material.

The PhD attendee, wants to find colleagues interested and working in the same field for cooperation and already posted paper for the same field for references or simply pose a question for given scientific problem and to open a discussion with participants sharing same interests.

The $\mathrm{X}$ mentor of the $\mathrm{Y} \mathrm{PhD}$ attendee wants to check if his delivered work is not a plagiat of any previous paper and works, already published in the network.

This and all a lot of scenarios are to be made possible with the support of the "Semantic Web" tools and methods [6].

\section{ONTOLOGY EXAMPLE}

The examples from the previous chapter give us clear hint of the needed ontology. From the first and the second example, comes out the ontology for the university members ( professor, student), faculty activities and study programs ( lectures, ETC credits, semester).

The third and the last scenario reveals the need for an ontology, covering the research interest, the PhD programe, the mentorship, plagiat and much more hidden subjects needing an definition of their existence and their relation with other things along a given business process and activity.

For the organization, where the faculty the departments and the employees and the lecturers are included, the ontology, would look like in the picture Figure 1 - Organisation ontology.

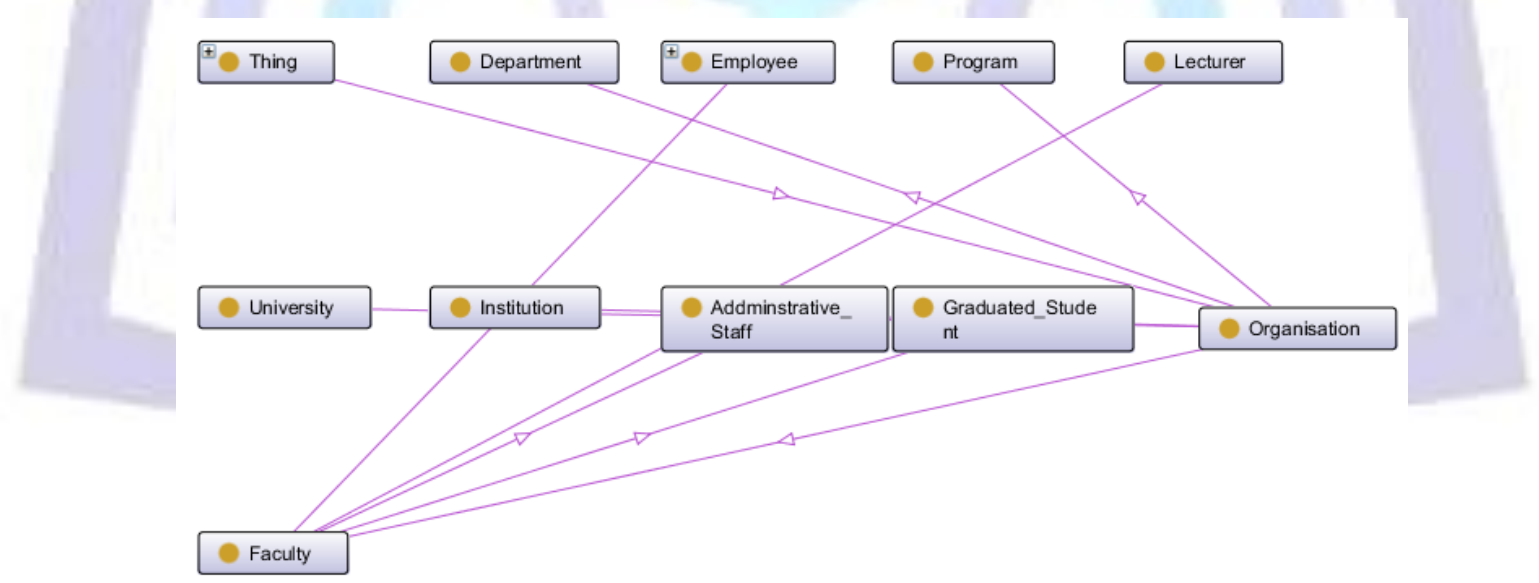

Figure 1 - Organisation ontology

The second part is the Social networks ontology. As for this kind of ontology's, there are plenty of open source projects, waiting to be exploited for this cause. One of the more reliable candidates for this meter is the "SIOC Initiative"[6] , were the SIOC acronym comes from the "Semantically-Interlinked Online Communities" and contains the needed ontology for interconnecting, or mapping the elements from our Social network, with the institution, and the Social networks, of the other institutions or more precisely universities with one and other, and thru that creating an endless knowledge management platform, all structured by help of ontology, providing us with an information, related to our interest.

Other helpful project being just as helpful as the above mentioned, is the FOAF Project, the "Friend Of A Friend". This project is equipped with the knowledge, the tools and the already defined ontology [7] for describing and interpreting the profiles on the Social Networks, and their relations and interactions between them, it's about the peoples. 


\section{THE RECIPES}

We have the ingredients, have the appetite for the knowledge and his management, we just need the recipe how to bring all that together under one hood.

Just for having clearer view, let just put the three ingredients on the table:

1. The social network

2. The users

3. The ontology

We need to take the materials published from the user in to the social network and map them to the defined ontology.

At this matter, we have two moments, the already existing published material and the future one, with constant update of eventual changes. The first moment is pretty difficult, as we know the heterogeneity of the information we put in our postings, blogs and so one. As for example, one student would name the lecture "Web Information Retrieval" the "WebIR", the other one would write its acronym "WIR" or the "Weblnf", and as because of this, the web information retrieval techniques as "Web Crawling" of the social networks, text processing of the content and trying to identify the entity, and map it to given ontology [8]. This approach can be semi automated, with an urgent need of manual revision and possible remapping in given cases.

As for the second mater, the phase "from now on", seems to be easier, because you will give the user the possibility of annotating its posts, and map it to its proper entity in the ontology [9]. Would it be easier? Does the user know the relevance, the benefits and the possibilities that the semantic web and its structured data makes possible and offers. Good start is by forcing them to give his posting an given "interest" (speak entity in our OWL) or to "TAG" it, trying to use the terms that are already in use on different blogs, social networks and other ways of mass communications, and not using the language like "is this Lecture of the type Faculty, and held by the entity Professor, which is sub entity of the Faculty", you will definitely scare them away. Work is almost done.

The last one in the list, the Ontology. Have we really covered everything with our predefined ontology or not give the user the possibility to add new entities to it?

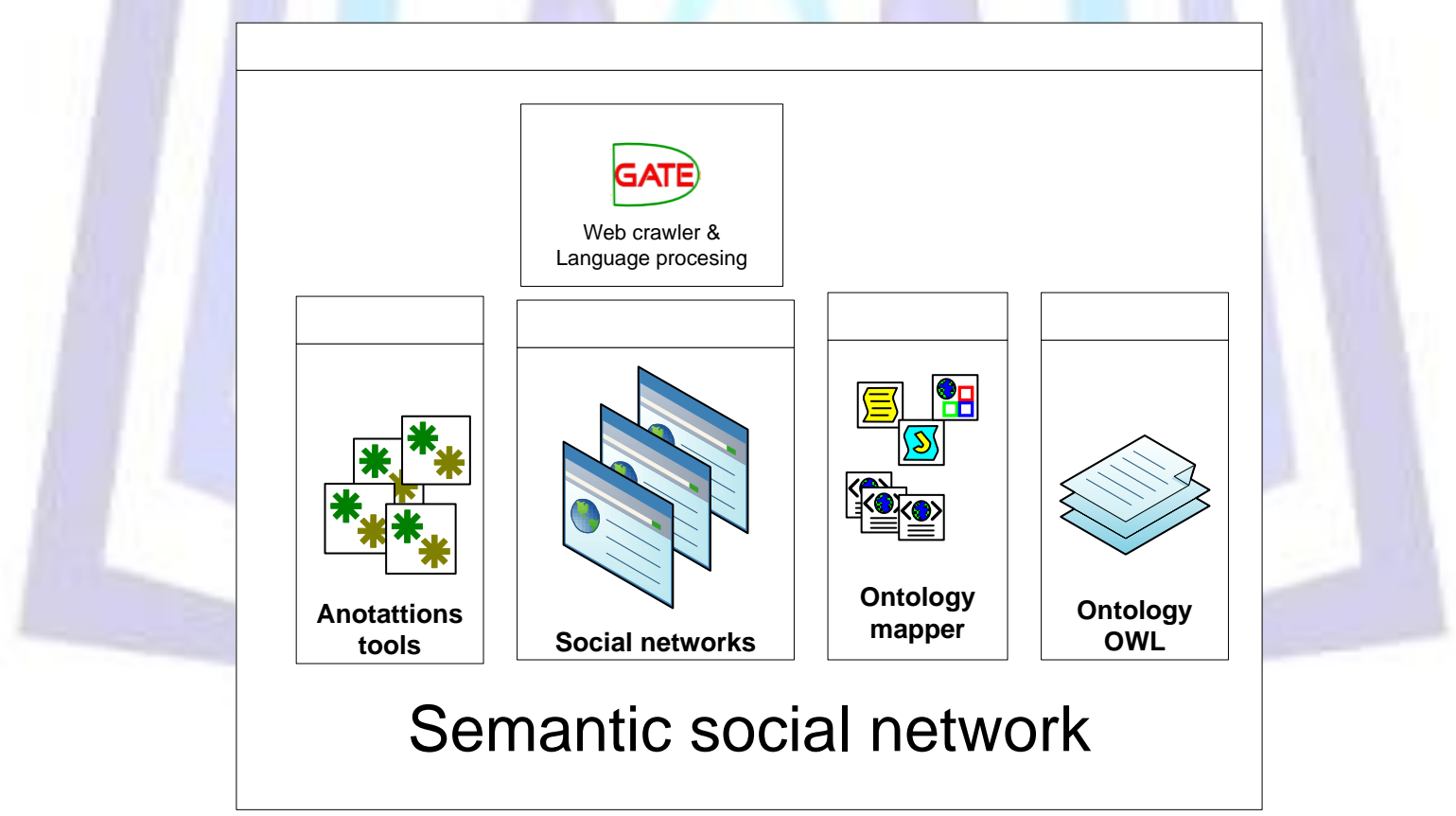

Figure 2 Semantic social network

The tools, the know-how is already there and also few existing semantic web systems like the "Oracle Spatial and Graph" or the "DBpedia", systems, that can serve like example, or like proof of the benefits we may have from the semantic web systems [10,11].

\section{CONCLUSION}

The dream of Tim Berners Lee [1] is (technically) possible. We have great network power, starting from the classic wired networks, up to Wireles and 3G,4G and WiMAX [13,14]. The computing power of the servers is not to be discussed , as we don't have to do with some enormous real time data processing or parallel computing, thing that are resolvable with the help of "Clod Computing", for just in case, if we consider face recognition functionality. 
Every time when someone starts to promote an product or to defend a cause with bringing all the "pro's" at once, be sure that there is an crucial "contra" argument. Related to our mater, the "semantic web", the crucial problem is the user itself and its unawareness of the benefits this system brings.

Make the user aware of its personal benefits and the benefits of the society from the structured data of the semantic web in general and its use for knowledge management. To achieve this goal, except for giving him free (free in price and no limitations whatsoever) access to considerable tools, and also prepare for him an existing structured data system that is in his or for group of users who can contribute to its development for brighter audience, until "all the world" is affected.

\section{REFERENCES}

[1]. The Semantic Web. Berners-Lee, Tim, Hendler, James and Lassila, Ora. 2001, Scientific American, p. 36.

[2]. W3C. Rdf Schema. [Online] 2004. [Cited: Korrik 22, 2014.] http://www.w3.org/TR/rdf-schema/.

[3]. OWL Web Ontology Language. OWL Web Ontology Language. [Online] 2003. http://www.w3.org/TR/2003/WD-owlguide-20030331/.

[4] Allemang, Dean and Hendler, James. Semantic Web for the Working Ontologist. s.I. : Elsevier Inc, 2011.

[5] A Translation Approach to Portable Ontology Specifications. Gruber, Thomas R. 1992, Knowledge System Laboratory Technical Report KSL 92-71, p. 24.

[6] The SIOC, initiative. The SIOC initiative (Semantically-Interlinked Online Communities). [Online] [Cited: Shtator 2, 2014.] http://sioc-project.org.

[7] SIOC-OWL. SIOC OWL. [Online] http://rdfs.org/sioc/ns\#.

[8] University of Sheffield. [Online] [Cited: October 20, 2014.] https://gate.ac.uk/.

[9] semanticweb.org. Semantic annotation tool. [Online] [Cited: October 20, 2014.]

http://semanticweb.org/wiki/Category:Semantic_annotation_tool.

[10].Oracle. Oracle Spatial and Graph. [Online]

http://www.oracle.com/technetwork/database/options/spatialandgraph/overview/index.html.

[11]. DBpedia. [Online] http://www.dbpedia.org/About.

[12]. Bhavani, Thuraisingam. XML databases and the Semantic WEB. s.I. : CRC Press, 2002.

[13]. Carrington, Peter J., Scott, John and Wasserman, Stanley. Models and Methods in Social Network Analysis.

[14]. Cross, Robert L. and Parker, Andrew. The Hidden Power of Social Networks: Understanding how Work Really Gets Done.

15. DuCharme, Bob. Learning SPARQL . s.I. : O'REILLY.

16. Elena, Simperl, Cuel, Roberta and Stein, Martin. Incentive-Centric Semantic Web Application Engineering. s.I. : Morgan \& Claypool Publishers, 2013.

17. Groppe, Sven. Data Management and Query Processing in Semantic Web Databases. s.I. : Springer verlag, 2011.

18. Levine, Martin D. Vision in man and machine. s.I. : McGraw-Hill, 1985.

19. Liyang, Yu. A Developer's Guide to the Semantic WEB. New York : Springer, 2011.

20. Mark, Watson. Practical Semantic Web and Linked Data Applications. 2011.

21. Martin, Kilduff and Wenpin, Tsai. Social Networks and Organizations. 2003.

22. Morvile, Peter and rosenfeld.Louis. Information Architecture for the World Wide Web: Designing Large-Scale Web Sites, 3rd Edition. s.I. : O'REILLY.

23. Parreiras, Fernando S. Semantic Web and Model-Driven Engineering. s.I. : Wiley-IEEE Press , 2012.

24. Passant, Alexandre. Semantic Web Technologies for Enterprise 2.0. s.I. : IOS Verlag, 2011.

25. Powers, Shelley. Practical RDF. s.I. : O'REILLY.

26. Raul, Garcia-Castro, et al. Workshop on Evaluation of Ontology-based Tools. EON 2008: Tenerife, Spain. [Online] [Cited: 1 14, 2014.] http://www.informatik.uni-trier.de/ ley/db/conf/eon/eon2008.html.

27. Youakim, Badr, Richard, Chbeir and Ajith, Abraham. Emergent Web Intelligence:Advanced Semantic Technologies. s.I. : Springer-Verlag London Limited, 2010.

28. The Semantic Web Revisited. Shadbolt, Nigel and Hall, Wendy. 2006, IEEE Computer Societ, pp. 96-101. 


\section{ISSN 2277-3061}

29. Ig Ibert, Bittencourt, et al. Research Directions on Semantic Web and Education. [Online] [Cited: Qershor 17, 2014.] www.ei.sanken.osaka-u.ac.jp/pub/isotani/semanticweb.pdf.

30. W3C. Extensible Markup Language (XML) 1.0 (Fifth Edition). [Online] Nëntor 26, 2008. [Cited: Qershor 18, 2014.] http://www.w3.org/TR/REC-xml/.

31. Antoniou, Grigoris and Van Harmelen, Frank. A Semantic Web Primer. London : The MIT Press, 2008.

32. W3C. W3C Semantic Web Activity. [Online] 2011. http://www.w3.org/2001/sw/.

33. W3C. RDFCore Working Group. [Online] Skurt 9, 2001. [Cited: Qershor 21, 2014.] http://www.w3.org/2001/sw/RDFCore/.

34. W3C. W3C Data Activity. [Online] Dhjetor 13, 2013. [Cited: Qershor 21, 2014.] http://www.w3.org/2013/data/ . 35. W3C. W3C. RDF/XML Syntax Specification. [Online] 2004. http://www.w3.org/TR/REC-rdf-syntax/.

36. Berners-Lee, T. Network Working Group. URI RFC. [Online] 2005. [Cited: Korrik 03, 2014.] http://www.rfceditor.org/rfc/rfc3986.txt. 